\title{
Moving Object Detection with High Precision via Marked Watershed and Image Morphology
}

\author{
Qingqing $\mathrm{Fu}^{1}$,Silin $\mathrm{Xu}^{2}$ and Aiping $\mathrm{Wu}^{3 *}$ \\ ${ }^{1,3}$ College of Electronics and Information Engineering, Yangtze University, \\ Jingzhou, Hubei, 434023, China \\ ${ }^{2}$ College of Meteorology and Oceanography, PLA Univ of Sci.\&Tech \\ 136820653@qq.com, ${ }^{2} X u$ Slin1111@sina.com, ${ }^{3}$ wuaping@yangtzeu.edu.cn
}

\begin{abstract}
This paper presents a non-stationary object detection method by exploring time-varying spatial domain information in full motion video. Initially, the edge maps of difference image between two adjacent frames and current frame is generated via the well-known Canny edge detector. The distance of the edge pixels between the difference image and the current video frame are confined within a small value to determine the initial edge mask for the object in motion. The horizontal and vertical filling followed by morphological opening and closing operator are applied on the initial edge mask to create initial temporal segmentation mask of the moving object. The morphological dilation and corrosion operator are utilized to obtain binary marker image of the foreground and background which are used to modify the multi-scale morphological gradient image of current frame. Finally, the watershed algorithm is performed on the modified gradients to find the non-stationary objects accurately in the spatial domain of motion frames. Processed video results show detection accuracy of $98 \%$ and $99 \%$ for four different video experimentation test-beds involving fast and slow human motion. In this operation, the proposed technique eliminates the problem of over-segmentation of the watershed algorithm and extracts visually distinct, contextually meaningful non-stationary objects as they randomly appear (or disappear) in video sequences.
\end{abstract}

Keywords: Video processing, multi-scale watershed gradient, horizontal and vertical filling, morphological operators, detection performance

\section{Introduction}

Temporal and spatial domain video analysis involves detecting and extracting objects in motion from video frame sequences. It plays an important role in video processing and compression in such application areas as various versions of MPEG and HP.264 [1], video surveillance, driver support, and video telecommunication. Although this field has experienced significant growth and matured considerably, it still poses challenging problems in real-time applications [2].

Over the last few decades, many researchers have proposed various video moving object segmentation techniques and algorithms [3-18], each of which has its own characteristics and special application platforms. The scheme presented in [3-5] makes use of the frame difference concept. The technique in [6] relies on the use of optical flow field. The background modeling principle is adopted for video segmentation in [7-8]. These algorithms take advantage of the time-domain information to obtain change detection mask, and then the post-processing is utilized on the mask to obtain a video object template. Although the efficiency of these techniques is high, the target

${ }^{*}$ Corresponding Author 
information may not be deduced with desired accuracy. In recent years, many segmentation schemes based on temporal and spatial information have been proposed [9-15], which take into account the spatio-temporal relationship and yield better partitioning results. As a spatial domain image segmentation algorithm with high performance, watershed algebraic methodology is incorporated into the video segmentation techniques based on the spatio-temporal information [9-14]. However, the watershed algorithm is particularly sensitive to noise, usually leads to over-segmentation. In [16], the segmentation is applied to the simplified image to reduce the influence of noise while preserving edge information. There can still be a considerable number of dark noisy points and profiles in the simplified image. In [12-13], the motion parameters estimated to merge the regions with coherent motions on the basis of watershed segmentation results. The region merging is carried out according to the similarity of the mean and standard deviation after the watershed segmentation in [11]. The technique described in [14] utilizes the Markov random field model for analysis and processing following the initial watershed segmentation. The watershed transformation usage in the mentioned literature is only confined in spatial partition followed by region merging which is time-consuming operation. The over-segmentation might still appear in some of these algorithms [12].

This paper aims to combine the temporal and spatial domain information for the purpose of video object segmentation in conjunction with the marked-watershed operation. In the proposed method, an initial edge mask is first derived in terms of the edge maps of difference image and current frame image. Temporal segmentation mask is then obtained in the time-domain making use of the horizontal and vertical filling. Comparing with the method in [17], which only uses edge maps of difference image to obtain the initial temporal mask, our approach yields more accurate initial temporal mask. Morphological techniques are applied to the initial temporal segmentation mask of the moving target to obtain the foreground and background markers for the watershed algorithm. This is utilized for guiding watershed segmentation in spatial image domain to obtain an accurate video object boundary. In turn, the overall approach effectively overcomes the problem of over-segmentation and avoids computationally expensive region merging process. As demonstrated in the experimental result section, the proposed method can segment and extract contextually significant and semantically meaningful objects in fast or slow motion. The overall algorithm has low computational complexity and execution time which is a high desirable factor in real time operation.

The remaining of this paper is organized as follows. An overview of the overall approach is discussed in the next section. The temporal segmentation is presented subsequently followed by the spatial domain partitioning and the experimental results. Discussion and conclusions are provided at the end.

\section{Description of the Overall Approach}

A block diagram representation of the proposed method is given in Figure 1. Our video segmentation algorithm starts with generating the difference image between the two adjacent frames. Because the frame difference image contains all the inter-frame change information with noise, the edge map of frame difference image is generated by the Canny operator which is known to have high accuracy in locating edges and strong ability in restraining the false edge points. From the same token of this argument, the current frame image is processed using the Canny operator. Initial edge mask for the object in motion is created via the edge maps of frame difference and the current frame image. Horizontal and vertical filling is followed by morphological operators, which are applied to the resultant edge mask to obtain the initial temporal segmentation mask of the moving target. Morphological procedures are applied to initial temporal segmentation mask to capture foreground and background markers for the watershed algorithm. In spatial 
domain segmentation, the problem of over-segmentation is solved, which are caused by noise or quantization error, via multi-scale morphological gradient operator [12]. Notice that the underlying operator has the ability in suppressing noise. For this reason, it is applied to the current frame image to create the gradient image. It is then modified by the previously obtained foreground and background markers. Watershed segmentation is performed on the modified gradient image in order to extract the moving objects. This means that the spatial segmentation is guided by the temporal segmentation results. At last, the performance of detection results is iteratively retained and effectively improved by the relevant assessment index and formula.

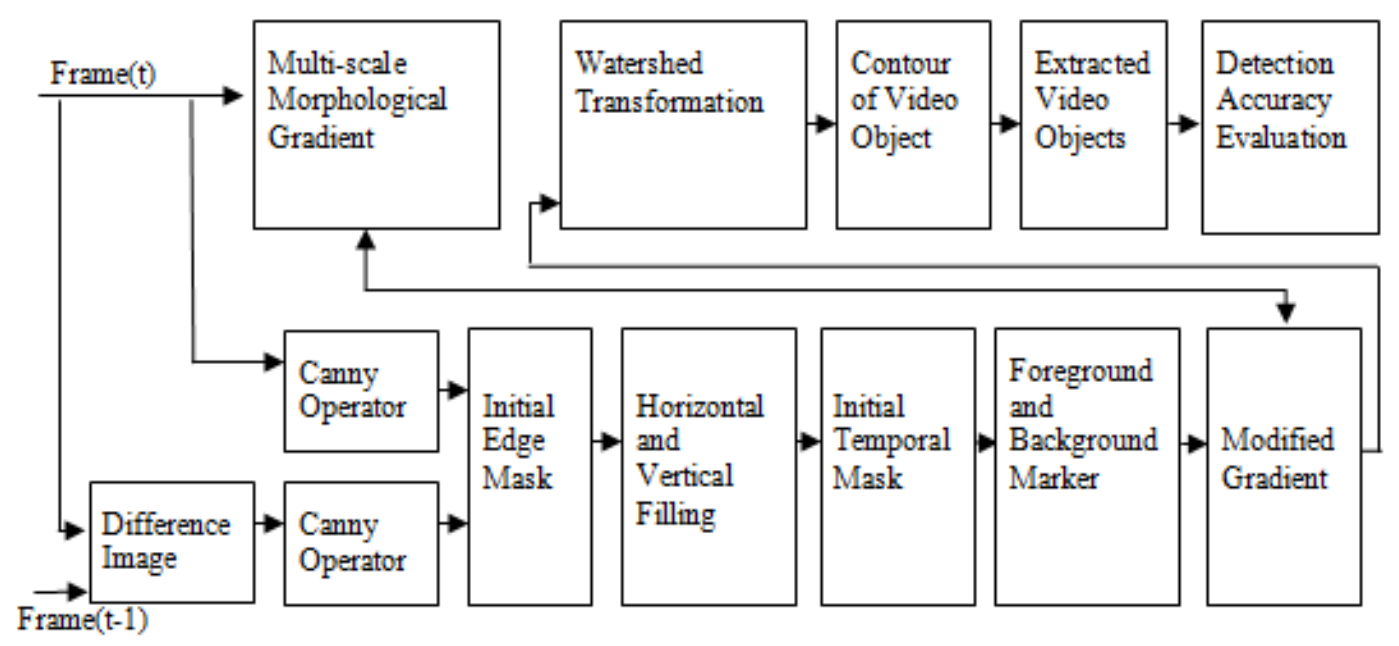

Figure 1. Block Diagram of the Proposed Algorithm

\section{Temporal Segmentation}

Change detection based on inter-frame difference is one of the most commonly used video image frame segmentation algorithms [3-5]. Temporal segmentation in the proposed scheme consists of two steps: The first step involves finding the frame difference image between the two adjacent frames and then applying the Canny operator on the frame difference and the current frame image to detect the edge information of the moving objects. The second step makes use of the horizontal filling, vertical filling and morphological post-processing technique on the initial edge map of the moving object so as to generate initial mask of the video object.

\subsection{Edge Detection of Moving Object}

Let $f_{t}$ denote the image of the current frame and $f_{t-1}$ represent the image of the previous frame. The edge of the inter-frame difference image $D E_{t}$ is defined as

$$
D E_{t}=\text { canny } \quad\left(\left|f_{t-1}-f_{t}\right|\right)
$$

Equation (1) dictates that the difference image edge map $D E_{t}$ is obtained by the Canny operator, which aims to optimize the signal-to-noise ratio with accurately positioning edge as proposed by Canny [18]. It is simple, high performance operator, which has been widely used in practice. Its edge detection operation can be described as follows: 1) The difference image between the current frame (number 62 in the selected example) and its adjacent frame in Figure 2, is the input image. As can be noticed, it suffers from great deal of noise even in stationary background. Note that if the object is in fast motion, the time interval of the adjacent frames is selected to be small. In the case of slow motion, the 
time interval should be longer. Then Gaussian low-pass filter with a standard deviation $\sigma$ is employed on the input image to reduce the influence of noise. 2) The local gradient (magnitude and direction) is computed using a gradient operator for each point in the smoothed image. 3) Appling the non-maximum value suppression (non-local maximum is set to 0 ) to the gradient magnitude to thin the detected edges. 4) The ridge pixels are then obtained using high and low double thresholds to detect and link edges. As a result, a map of clear and thinned edges is reached for further utilization (see Figure 3). In the same way, the edge map $C E_{t}$ of the current frame image can be generated (see Figure.4). The coarse moving edge map produced is given by selecting all edge pixels belonging to the $C E_{t}$ within a small distance $\mathrm{T}$ of the edge pixels belonging to the $D E_{t}$. The coarse moving edge map, denoted by $M E_{t}$, is defined as

$$
M E_{t}=\left\{y \in C E_{t}\left|\min _{x \in D E_{t}}\right| x-y \mid \leq T\right\}
$$
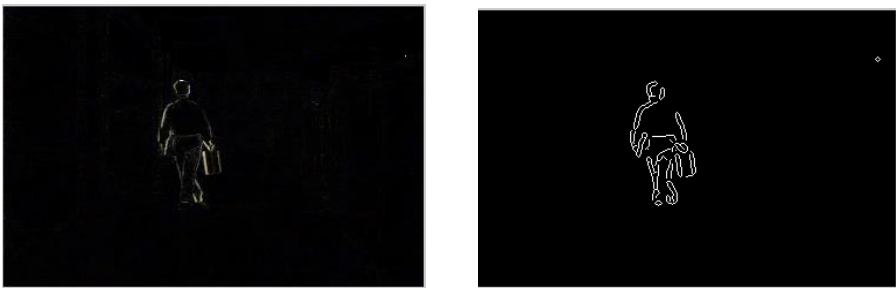

\section{Figure 2. Difference Image Figure 3. Edge Map of Difference Image}

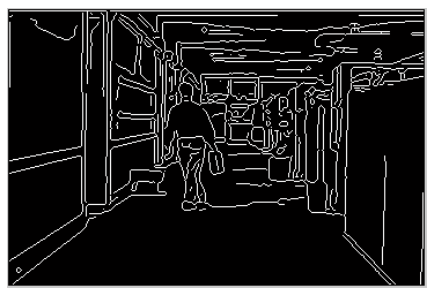

Figure 4. Edge Map of Current Frame

\subsection{Extraction of Moving Object Mask}

After the coarse moving edge map $M E_{t}$ is created, the initial edge mask (see Figure 5,) can be obtained by removing all the connected components that have fewer than those pixels from the map $M E_{t}$. The initial mask of moving object is formed using the horizontal and vertical filling. This operation proceeds by filling the region between the first and last edge points in each row and the vertical for each column of the initial edge mask. In this way, both horizontal and vertical candidates for the moving object are located correctly. Intersection regions are the coarse moving object mask through logical AND operation between the horizontal and vertical filling map. Figure.6 shows the results of the horizontal and vertical filling of the walking person profile in Figure 5.

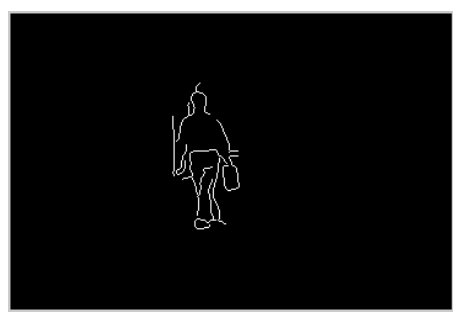

Figure 5. Initial Edge Mask

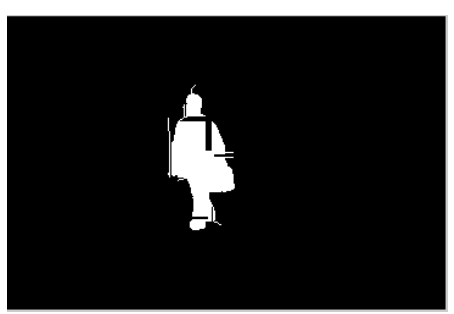

Figure 6. Coarse Moving Object Mask

Due to the influence of the noise and the discontinuous edge in the initial edge mask, the coarse moving object mask may have incomplete and redundant part. Hence, the intersection regions are further processed by morphological operations as explained below. The results yielded are included in Section 5 .

The dilation and erosion are the most fundamental operations in morphological image processing. Suppose that $\mathrm{A}$ and $\mathrm{B}$ be sets in $Z^{2}, B$ is used as structuring elements the 
dilation of $A$ by $B$, denoted by $A \oplus B$, Corrosion of $A$ by $B$, denoted by $A \Theta B[18]$.

Opening and closing are two other important morphological operations used in this work. The opening of the set $\mathrm{A}$ by the structuring element $\mathrm{B}$, denoted as $A \circ B$, is given by [18]

$$
A \circ B=(A \Theta B) \oplus B
$$

Opening smoothes the contour of an object and eliminates thin protrusions. Similarly, the closing of the set $\mathrm{A}$ by the structuring element $\mathrm{B}$, denoted with $A \bullet B$, is defined as [18]

$$
A \bullet B=(A \oplus B) \Theta B
$$

Closing also tends to smooth sections of contours, removes small holes, and fills gaps in the contour.

According to the above described morphological processing framework, the opening operator is first used on the coarse moving object mask and eliminates thin and redundant parts. Then, the MatLab function 'bwareaopen' is employed to remove all the connected components that have fewer points than a certain number of pixels so as to eliminate the background noise. At last, the long thin gaps are filled in the contour using closing operator, so as to create an initial binary mask of the moving object. In the above mentioned morphological operator, a flat, disk-shaped structuring element is selected, which can eliminate dependence of the gradient edge on the edge direction.

\section{Spatial Segmentation}

Coarse area is only obtained through temporal segmentation due to the complexity of the motion information. Spatial segmentation is needed to get more accurate object boundary. Watershed algorithm proposed by Vincent based on immersion simulation [19] is known to be a fast segmentation method of the mathematical morphology in the field of image segmentation. An image is often interpreted as geographical surfaces and its gray level is regarded as altitude. This can be explained further with the aid of Figure 7, which shows a simple topographic view, a local minimum corresponds to the valley, whereas the maximum corresponds to the peak. Water will overflow upward from each local minimum and different local area will be gradually filled with water. As the water continues to rise, the rising water in various regions is about to merge. If a dam is built at the meeting place to prevent the merging, then the topography is divided into different regions, which are called the catchment basins. At the end of this immersion procedure, each minimum is completely surrounded by dams, the edge of the region where dam is built called watershed. Hence, watershed segmentation is to find catchment basins and watershed ridge lines.

Watershed algorithm is usually performed on the gradient image [18]. Conventional gradient operators generally produce many local minima resulting in over-segmentation, which is caused by noise or quantization error. To alleviate this problem and reduce the morphological gradient effect on abrupt changes in input image, morphological gradient image is treated by symmetrical structuring elements which have less dependence on edge direction. In this paper, the multi-scale morphological gradient algorithm proposed by Wang in [12] is applied to the current video frame, and foreground and background markers are used to control watershed partitioning to achieve better spatial segmentation. 


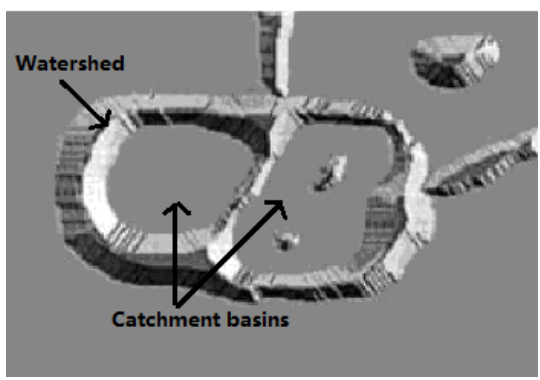

Figure 7. Modeling of Contours by Watershed Transform

\subsection{Multi-Scale Morphological Gradient}

Simple single-scale morphological gradient operator is defined as

$$
G(f)=f \oplus B-f \Theta B
$$

where $f$ denotes the input gray scale image, $B$ is the structuring element, and the symbols $\oplus$ and $\Theta$ denote the morphological dilation and erosion operations mentioned in Section 3.2. Its performance depends on the size of the structuring element B. if B is large, it will cause the overlap between the edges which may lead to the gradient maxima not coinciding with edges. However, if the structuring element is too small, this gradient operator has a high spatial resolution and produces a low output value for ramp edges. The multi-scale morphological gradient operator is described in [12]

$$
M G(f)=\frac{1}{3} \sum_{i=1}^{3}\left[\left(\left(f \oplus B_{i}\right)-\left(f \Theta B_{i}\right)\right) \Theta B_{i-1}\right]
$$

where $B_{i}$ is a group of disk-shaped structuring element and its radius is $2 i+1,0 \leq i \leq 3$. In this definition, the multi-scale morphological gradient has respective advantages of both large and small structuring elements. It is more robust to noise and edge interaction due to the averaging operation used in the algorithm. It effectively enhances blurred edges and reduces the number of the irrelevant local minima.

\subsection{Marker Extraction}

Marking the object in the watershed transformation is very important in the control of the over-segmentation. Markers are connected component belonging to an image. If we can find a marker set of the foreground completely included in the objects of interest, and a marker set of the background completely included in the region of background, these markers can be used to modify the gradient image so that the local minimum area appears only in the marked location.

After using the erosion and dilation operator with the size of $\mathrm{r} 1$ and $\mathrm{r} 2$ on the initial binary mask of the moving object mentioned in Section 3.2, we can obtain binary image f1 and $\mathrm{f} 2$, the region with value of 1 in the image $\mathrm{f} 1$ belongs to the set of the objects' regions, which will be regarded as foreground marker. Thus, $\mathrm{f} 1$ represents the foreground mark image. Assume that image $\mathrm{fb}$ is the complement of the image $\mathrm{f} 2$. Then the region with value of 1 in the image fb belongs to the set of the background region, which will be considered as background marker. Thus, fb denotes the background marked image. The area between the foreground and background marker is contour region, in which watershed algorithm can find the dividing line between the foreground and background regions. This is referred to as the contour of the object. The union of $\mathrm{fl}$ and fb is used to modify multi-scale morphological gradient of the current frame. Here, the Gmark is denoted as the modified gradient image, the MatLab function 'imimposemin ' is used to find the minima imposition so as to make the local minimum area only appears in the 
marked position. Implementation syntax of this function is of the form

Gmark =imimposemin(MG(f),f1|fb)

where $\operatorname{MG}(\mathrm{f})$ is the multi-scale morphological gradient of current image. At last, watershed segmentation algorithm will perform on Gmark and the MatLab function 'watershed' is employed to find watershed ridge line which is also called the ideal video object contour. Implementation of this function is described as in (8), where contour denotes watershed ridge line.

contour=wartershed(Gmark)

Marker process is applied to traditional watershed algorithm, and over-segmentation phenomenon does not appear during the segmentation phase. There is no need to conduct region merging. In this way, the computational complexity is reduced considerably.

\section{Experimental Results}

The algorithm proposed in this paper for video segmentation is implemented using the MatLab software of version R2008a. The selected test sequences are the standard video sequences called hall_monitor and mother\&daughter in the filed of the image segmentation, and compared with the results of the watershed algorithm directly used on gradient image. The segmentation process of sequence hall_monitor is illustrated in Figures 8,9 and10. The sequence hall_monitor exhibits rapid and great deformable motion over the stationary background but more complex textures. Further, the number of the motion objects in the scene changes from a single subject to multiple individuals. It also exhibits illumination effect and the resulting visualization. Figure $8(a)$, is the original frame 62. Figure 8(b), is initial edge mask resulting from the Canny operator on the current frame and the frame difference image between the frame number 62 and its adjacent image. Figure 8(c), shows the result of the initial temporal mask after horizontal filling, vertical filling and morphological operations applied to Figure 8(b). Figure 8(d), is the result obtained by using the watershed segmentation method on the gradient image of the current frame. Notice that over-segmentation problem is considerably serious for the object extraction operation. Figure 8(e), displays the contour image after executing watershed transform on the modified gradient image. The extracted non-stationary video object is shown in Figure 8(f). Figure 9(a-f), is the extraction process of the frame 226 of the sequence hall_monitor. As seen in Figure 9, the method can segment two moving objects in the video. Figure 10(a-f), are the segmentation results of the frame 292 of the sequence hall_monitor. It is noticeable that the algorithm has a good performance on detecting disappearance of existing objects. Figure 11(a-f), are the segmentation results of the frame 15 of the mother\&daughter video sequence. The clip mother\&daughter is a typical video-conference scene that exhibits slow and smooth motion over a stationary and complex texture background. The head of the mother has relatively large motion while the chest part exhibits small motion. It can also be noticed that the whole chest of the daughter presents little motion through the entire video sequence. The segmentation results of the above two video sequences show that the proposed algorithm finds foreground and background markers through the temporal segmentation information, which are used to control the spatial segmentation and effectively overcomes the over-segmentation problem. Further, it can segment and extract meaningful single and multiple objects with fast and slow motion, which may be newly appearing or disappearing in video frame sequences. 

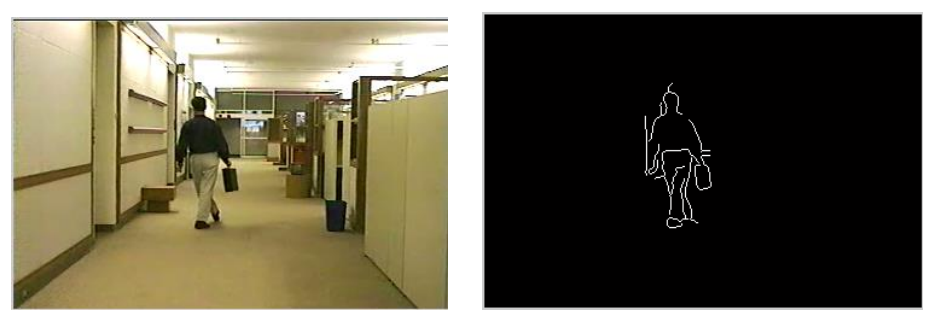

(a) original frame 62

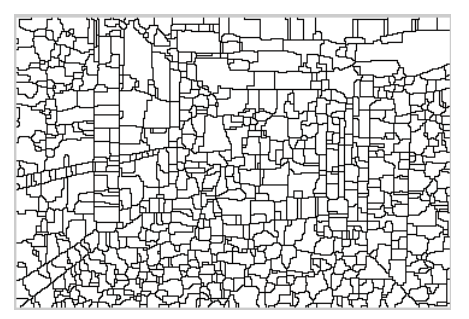

(d) Segmentation results directly using the watershed (b) initial edge mask

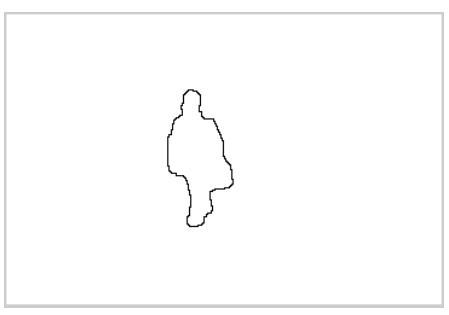

(e) contour image of the marked-watershed

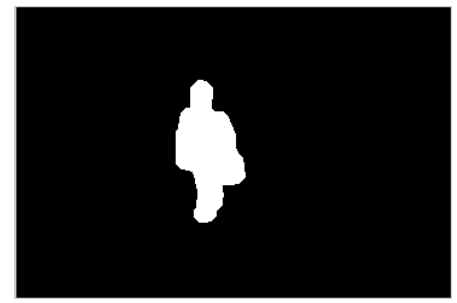

(c) initial temporal mask

Figure 8. Segmentation Results for the Video Sequence Hall_monitor(62)

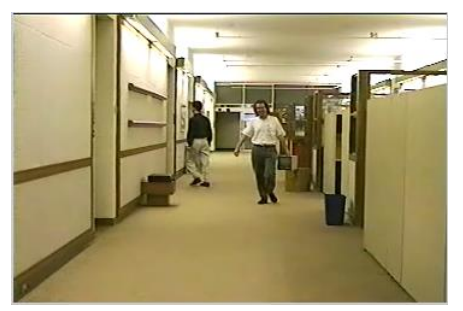

(a) original frame 226

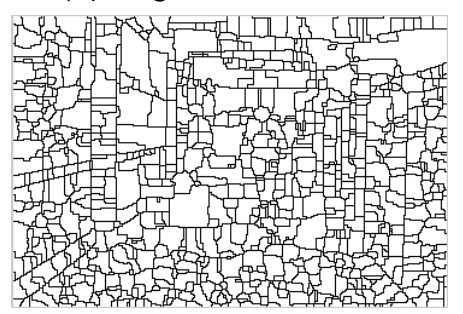

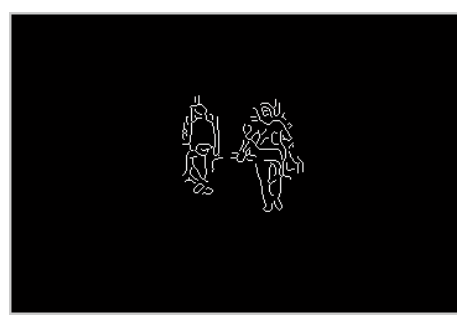

(b) initial edge mask

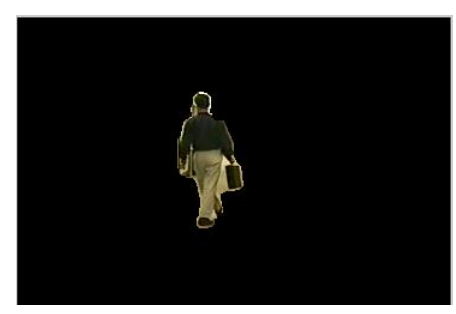

(f) extracted video motion object

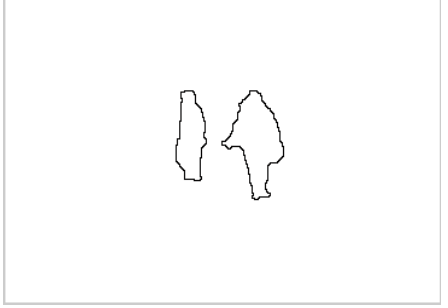

(e) contour image of the marked-watershed

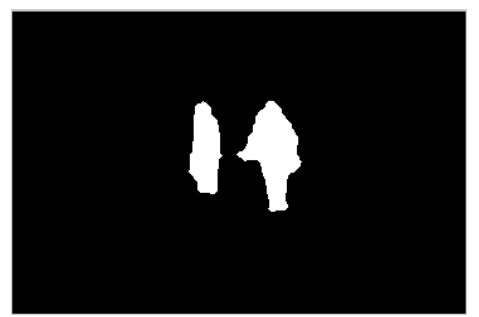

(c) initial temporal mask

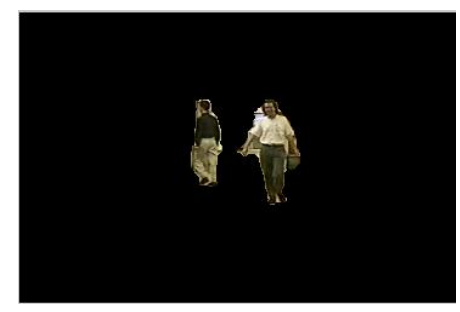

(f) extracted video motion object

using the watershed

Figure 9. Segmentation Results for the Video Sequence Hall_monitor(226)

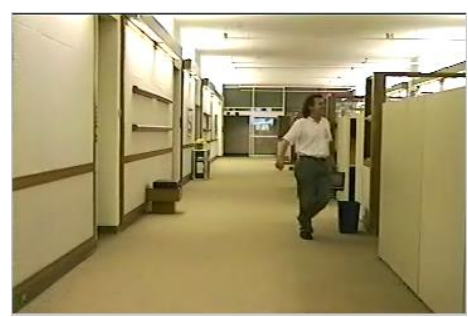

(a) original frame 292

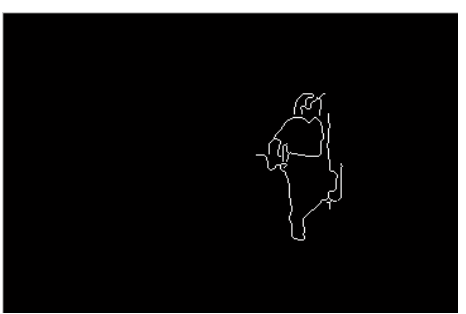

(b) initial edge mask

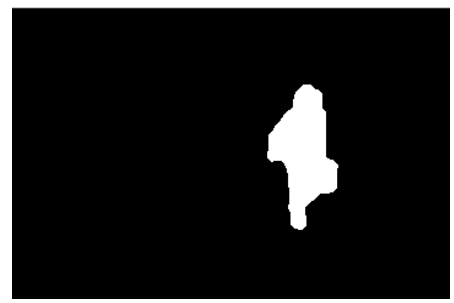

(c) initial temporal mask 


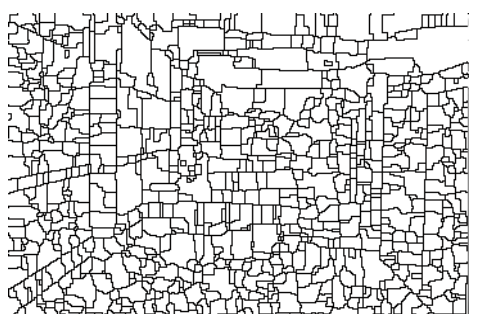

(d) Segmentation results directly using the watershed

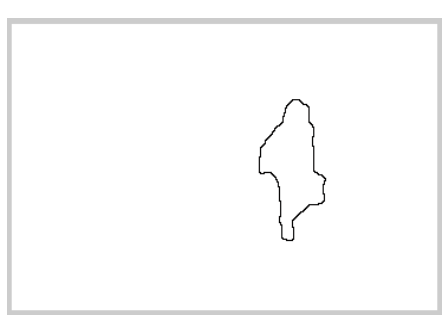

(e) contour image of the marked-watershed

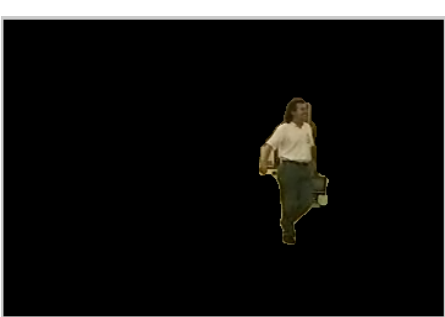

(f) extracted video motion object

\section{Figure 10. Segmentation Results for the Video Sequence Hall_monitor(292)}

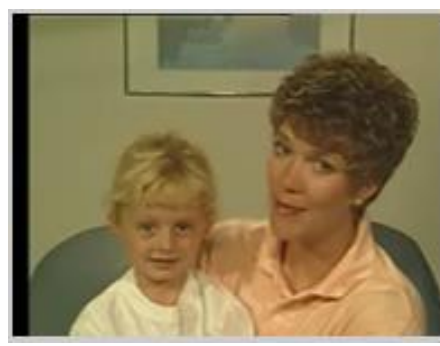

(a) original frame 15

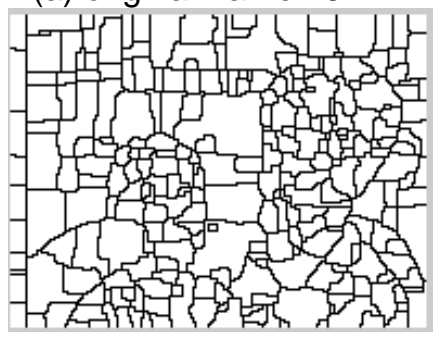

(d) Segmentation results directly using the watershed

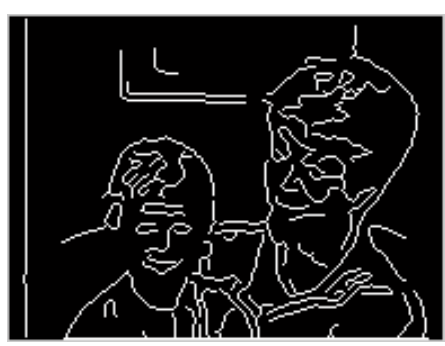

(b) initial edge mask

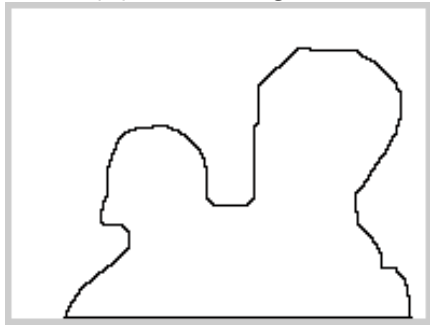

(e) contour image of the marked-watershed

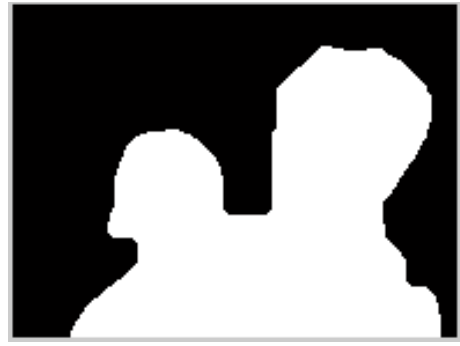

(c) initial temporal mask

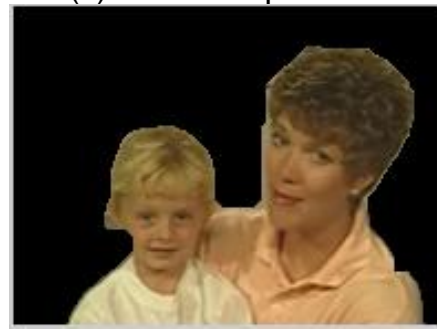

(f) extracted video motion object

\section{Figure 11. Segmentation Results for the Video Sequence mother\&daughter}

To evaluate the performance of our segmentation method objectively, comparing the machine segmentation (MS) results with its corresponding hand labeled ground truth (GT) image. The performance is measured as follows. Any pixel which is extracted as object in both MS and GT is defined as a true positive (TP). Any pixel which is extracted as object in MS but not in GT is defined as a false positive (FP). Any pixel which is extracted as non-object both in MS and GT is defined as a true negative (TN). Any pixel which is extracted as object in GT but not in MS is defined as a false negative (FN). The true positive rate (TPR) and The false positive rate (FPR) can be defined as [20].

$T P R=\frac{\text { number of } T P}{\text { number of } T P+\text { number of } F N}$

$F P R=\frac{\text { number of } F P}{\text { number of } F P+\text { number of } T N}$

A measurement of accuracy (AC) can be determined via [20]

$A C=\frac{\text { number of } T P+\text { number of } T N}{\text { number of }(T P+T N+F P+F N)}$

These performance parameters describe the detection accuracy of the moving object. 
For a perfect segmentation, values of TPR and AC should be 1, whereas for FPR should be 0 . Table 1 shows the values of the TPR, FPR, and AC for the above test sequences. The values of the parameters show that the true positive rate and accuracy of proposed algorithm are high and the false positive rate is low, which indicates that the proposed algorithm produces accurate segmentation and, consequently, reliable non-stationary object detection results.

Table 1.The TPR, FPR and AC of Test Sequence

\begin{tabular}{|l|l|l|l|}
\hline \multirow{2}{*}{ Sequence Name } & \multicolumn{3}{|c|}{ Parameters } \\
\cline { 2 - 4 } & TPR & FPR & AC \\
\hline hall_monitor(62) & 0.9443 & 0.0054 & 0.9927 \\
\hline hall_monitor(226) & 0.9554 & 0.0056 & 0.9929 \\
\hline hall_monitor(292) & 0.9915 & 0.0062 & 0.9937 \\
\hline Mother\&daughter(15) & 0.9914 & 0.0241 & 0.9829 \\
\hline
\end{tabular}

\section{Conclusions}

In this paper, a segmentation algorithm of moving objects in video sequence is proposed based on temporal and spatial domain information which makes use of the marked-watershed procedure. During the temporal segmentation phase, the Canny operator is employed to detect the edges of frame difference between the two adjacent frames and current frame image. We impose restriction on the distance of the edge points between the difference image and current frame image within a small value in order to determine the initial edge mask for the object in motion. The horizontal and vertical filling is applied to the initial edge mask and morphological techniques are further used to obtain initial temporal segmentation mask of the moving object. Erosion and dilation operation is chosen to work on the temporal segmentation mask to extract foreground and background markers for the watershed algorithm. In the spatial segmentation phase, multi-scale morphological gradient operator with strong ability in suppressing noise is applied to the current frame to produce the gradient image, which is then modified by the foreground and background markers. At last, watershed segmentation is performed on the modified gradient image. The proposed technique can efficiently segment and extract meaningful single and multiple moving objects with slow or fast motion, which may be newly appearing in the video sequence with stationary background of complex textures. The method appears to be least influenced by the noise and illumination variation. It restrains efficiently the phenomenon of the over-segmentation of the watershed algorithm and avoids region merging process in order to reduce the computational complexity. We evaluate the performance of the proposed segmentation method objectively. Test results show the detection accuracy of $98 \%$ and $99 \%$ for three different video experimentation sequences. The proposed technique involves several parameters such as the high and low thresholds in the Canny operator, the size of the structuring element and the small distance in determining the initial edge mask which are empirically set in the simulations. The future extension of this work is toward automatic determination of the parameters used in this technique and detection of objects with part of occlusion in motion.

\section{Acknowledgment}

This work has been funded by The National Natural Science Foundation of China (No. 51541408) and the Education Department of Hubei Province, China (D20141303).

\section{References}

[1] T. Sikora, "The MPEG-4 video standard verification model”, IEEE Transactions on Circuits System for Video Technology, vol.7, (1997), pp.19-31. 
[2] K. N.Ngan and H. Li, "Video segmentation and its applications [electronic resource]" , New York : Springer, (2011).

[3] NeriA, Colonnese S, Russo G and Talone P, "Automati moving object and background separation", SignalProcessing,APR, vol. 66, no. 2, (1998), pp. 219-32.

[4] C. Kim and J. N. Hwang, "Fast and Automatic Video Object Segmentation and Tracking for Content-Based Application”, IEEE Transactions on Circuits \& Systems for Video Technology Vol. 12, Issue 2, (2002) ,p.122-29.

[5] X. Hu and W. Hu, "New Video Image Segmentation Algorithm", Computer Engineering,36(23), (2012),p.217-19.

[6] J. Barron, D. Fleet and S. Beauchemin, "Performance of optical flow techniques", International Computer Vision12(1), (1994),p.42-77.

[7] D. W. Chinchkhede and N. J. Uke, "Image Segmentation in Video Sequences Using Modified Background Subtraction", International Journal of Computer Science \& Information Technology Vol. 4, Issue 1, (2012) , p.93-104.

[8] L. F. Teixeira, J. S. Cardoso and L. C. Real, "Object Segmentation Using Background Modelling and Cascaded Change Detection", Journal of Multimedia, vol. 2, no. 5, (2007), pp. 55-65.

[9] R. Li, S.Yu, and X. Yang, "Efficient Spatio-temporal Segmentation for Extracting Moving Objects in video Sequences", IEEE Transactions on Consumer Electro nics, vol. 53, no. 3, (2007), pp. 1161-67.

[10] R. LI, S. Y. Yu and X. Wang, "Unsupervised Spatio-Temporal Segmentation for Extracting Moving Objects in Video Sequences", J. Shanghai Jiaotong Univ. (Sci.), vol. 14, no. 2, (2009), pp. 154-61.

[11] Q. Deng, H. Liu and L. Wu, "Video Object Segmentation Algorithm Based on Watershed and Region Merging",Modern Scientific Instruments, no. 1, (2010),p.34-38.

[12] D. Wang, "Unsupervised video segmentation based on watersheds and temporal tracking", IEEE Transactions on Circuits and Systems for Video Technology Vol. 8, (1998), pp.539-46 .

[13] K. Ganesan and S. Jalla, "Video Object Extraction Based on a Comparative Study of Efficient Edge Detection Technique" ,International Arab Journal of Information Technology (IAJIT), vol. 6, no. 2, (2009), pp.107-15.

[14] I. Patras, E. A. Hendriks and R. L. Lagendijk, "Video segmentation by MAP labeling of watershed segments", IEEE Trans Pattern Anal Machine Intell, vol. 23, (2001), pp.326-32.

[15] J. Wang, Z. H. Li, D. M. Li and H. Sun, "A spatio-temporal video segmentation method based on edge information", International Conference on Energy Systems and Electrical Power (ESEP 2011), Energy Procedia, vol. 13, (2011), pp. 5508-15.

[16] G. Hai, S. Wan, C. Hou, and C. Huan, "Improved techniques for automatic image segmentation”, IEEE Transactions on Circuits and Systems for video technology, vol. 11, no. 12, (2001), pp. 1273 - 80

[17] Q. Fu and M. Celenk, "Spatio-Temporal Video Motion Detection Using Marked-Watershed and Morphological Operations",Journal of Communication and Computer, vol. 10, (2013), pp. 1181-89.

[18] R. C. Gonzalez, R. E. Woods and S. L. Eddins, "Digital Image Processing Using Matlab", Pearson Prentice-Hall, Upper Saddle River, NJ, USA, (2003).

[19] L. Vincent and P. Soille, "Watersheds in digital spaces: An efficient algorithm based on immersion simulations", IEEE Trans. on Pattern Analysis and Machine Intelligence, vol. 13, (1991), pp. 583-98.

[20] D. M. W. POWERS, "Evaluation, From Precision, Recall and F-measure to Roc,Informedness, Markedness \& Correlation”, Journal of Machine Learning Technologies, vol. 2, no. 1 , (2011), pp. 37-63.

\section{Authors}

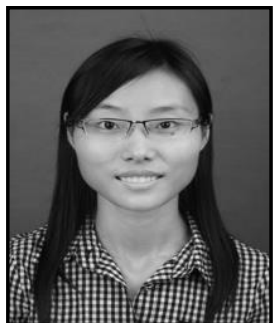

Qingqing Fu, she received the B.S. and M.S. degree from Department of Electronics and Information Engineering, Yangtze University in China, in 2001 and 2004, respectively. Her research interests include image processing, video segmentation and digital electronic techniques.

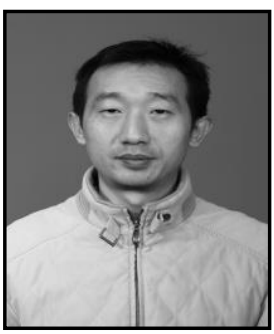

Silin $\mathrm{Xu}$, he received the B.S. and M.S. degree from the school of Meteorology and oceanography, PLA University of Science and Technology in 2000 and 2003, respectively. His research interests are information and signal processing. 


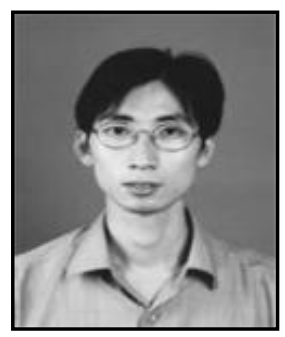

Aiping Wu, he obtained his Ph.D. Degree in China University of Geosciences (Wuhan) in 2013, the B.S. and M.S. degrees from Yangtze University, in 2000 and 2006, respectively. Now he is an associate professor in Yangtze University. His primary scientific interests are in the method of geophysical instrument and signal processing. 